\title{
VARIATIONAL FORMULAS ON LIPSCHITZ DOMAINS
}

\author{
ALAN R. ELCRAT AND KENNETH G. MILLER
}

\begin{abstract}
A rigorous treatment is given of variational formulas for solutions of certain Dirichlet problems for the Laplace operator on Lipschitz domains under interior variations. In particular we extend well-known variational formulas for the torsional rigidity and for capacity from the class of $C^{1}$ domains to the class of Lipschitz domains. A motivation for this work comes from the use of variational methods in the study of Prandtl-Batchelor flows in fluid mechanics.
\end{abstract}

\section{INTRODUCTION}

The problem of differentiating domain functionals, functionals depending on a variable domain $\Omega \subset \mathbf{R}^{n}$, is a problem with many applications and one which has a history going back at least to Rayleigh [14] and Hadamard [9]. The problem was investigated rigorously and extensively over forty years ago by Garabedian, Schiffer and others [8]. In particular the method of interior variations is used in [8] to give a rigorous proof of Hadamard's variational formula for the Green's function, the proof being valid for domains which are sufficiently regular to apply the divergence theorem. This includes Lipschitz domains. Variational formulas for many domain functionals can be found formally by integrating the Hadamard formula with respect to one of the variables. However, care must be exercised in so doing, as the Hadamard formula is an asymptotic formula and the $o(\varepsilon)$ remainder term is not uniform over the entire domain in either variable. Alternatively, by making use of the implicit function theorem, Simon [15] has given a rigorous treatment of differentiation of certain domain functionals that avoids using the Hadamard formula. His proof, however, requires that the domains have regular boundary.

The class of Lipschitz domains seems to be an appropriate class in which to consider many variational formulas. Our proofs will make use of a formula of Garabedian and Schiffer for the variation of the Green's function, together with results by Dahlberg [7], Jerison and Kenig [11] giving estimates for solutions of the Dirichlet problem on Lipschitz domains.

We consider here only the interior variations of Garabedian and Schiffer (we allow variations by Lipschitz continuous vector fields $S$ rather than $C^{3}$ vector fields), although other types of variations have been investigated. For example, in [10], Jerison has considered the variation of the capacity of a convex domain

Received by the editors June 21, 1994; originally communicated to the Proceedings of the AMS by J. Marshall Ash.

1991 Mathematics Subject Classification. Primary 35J20, 49J50; Secondary 76C05.

The authors were partially supported by NSF-Epscor grant OSR-9255223. 
$D$ under variations in terms of Minkowski sums $D_{t}=(1-t) D+t D_{1}$. One might also consider perturbations which arise from smoothing a Lipschitz boundary as in [11, p. 28]. Such variations do not fit into the framework of interior variations, however, and require a different approach.

A compactness result of Chenais [6] shows that the class of Lipschitz domains may be an appropriate setting for optimization of domain functionals. Our interest in variational formulas on Lipschitz domains arose out of an attempt to use these results to give a rigorous proof of the existence of Prandtl-Batchelor flows in fluid mechanics. An existence result for these flows is known to be an elusive problem (Caflisch [4, p. 16]) and we also were not entirely successful. A brief discussion of our approach to the Prandtl-Batchelor problem is given in the last section.

\section{RESUlts}

We restrict our attention to bounded domains $D$ in $\mathbf{R}^{n} . D$ is Lipschitz if for each $z \in \partial D$ there is a coordinate system $\left(x^{\prime}, x_{n}\right)$ in $\mathbf{R}^{n}=\mathbf{R}^{n-1} \times \mathbf{R}$, a neighborhood $U=U_{1}(z) \times U_{2}(z)$ and a Lipschitz function $\psi_{z}$ such that $U \cap D=\left\{\left(x^{\prime}, x_{n}\right) \in U: x_{n}>\psi_{z}\left(x^{\prime}\right)\right\}$. A constant $C$ such that $\left|\psi_{z}(t)-\psi_{z}(s)\right| \leq$ $C|t-s|$ for all $z \in \partial D, t \in U_{1}(z), s \in U_{1}(z)$ is called a Lipschitz constant for $D$.

We consider first the behavior of solutions of the Dirichlet problem $-\Delta u=f$ on $D, u=0$ on $\partial D$, under variations of the domain $D$ using the method of interior variations (Garabedian and Schiffer [8]), where $f$ is an $L^{2}$ function defined in a neighborhood $D^{\prime}$ of the closure of $D$. Let $S$ be a Lipschitz continuous vector field defined on $D^{\prime}$. We can choose $\varepsilon_{0}>0$ sufficiently small that for $|\varepsilon|<\varepsilon_{0}$ the transformation $\phi_{\varepsilon}(x)=x+\varepsilon S(x)$ maps $D$ homeomorphically onto a Lipschitz domain $D_{\varepsilon} \subset D^{\prime}$. Let $u_{\varepsilon}$ satisfy $-\Delta u_{\varepsilon}=f$ on $D_{\varepsilon}, u_{\varepsilon}=0$ on $\partial D_{\varepsilon}$ and let $u(x, \varepsilon)=u_{\varepsilon}\left(\phi_{\varepsilon}(x)\right)$. Since $D$ is Lipschitz and $\frac{\partial u_{0}}{\partial n} \in L^{2}(\partial D)$, there is [11] a solution $u^{\prime}$ to the Dirichlet problem

$$
\Delta u^{\prime}=0 \text { on } D, \quad u^{\prime}=-S \cdot \nabla u_{0} \text { on } \partial D .
$$

Theorem 1. For sufficiently small $\varepsilon_{0}>0$ the function $\varepsilon \mapsto u(\cdot, \varepsilon)$ from $\left(-\varepsilon_{0}, \varepsilon_{0}\right)$ into $H_{0}^{1}(D)$ is differentiable and

$$
\left.\frac{d u(\cdot, \varepsilon)}{d \varepsilon}\right|_{\varepsilon=0}=u^{\prime}+S \cdot \nabla u_{0} .
$$

Here $H_{0}^{m}(D)$ denotes the closure of $C_{0}^{\infty}(D)$ in $H^{m}(D)$ where $H^{m}(D)$ is the Sobolev space $H^{m, 2}(D)$. The theorem will be proved in Section 2. A consequence of this result is a variational formula for the torsional rigidity of $D$ where $D$ is a bounded Lipschitz domain in $\mathbf{R}^{n}$ bounded by a simple closed surface $\Gamma$. The torsional rigidity of $D$ is defined by $T(D)=\int_{D}|\nabla u|^{2} d x$ where $u$ is defined by $-\Delta u=2$ on $D, u=0$ on $\partial D$.

Corollary 2. Let $D$ be a bounded Lipschitz domain in $\mathbf{R}^{n}$ bounded by a simple closed surface $\Gamma$. Then

$$
\left.\frac{d T\left(D_{\varepsilon}\right)}{d \varepsilon}\right|_{\varepsilon=0}=\int_{\Gamma}\left|\nabla u_{0}\right|^{2}(S \cdot n) d s
$$

where $n$ is the outward normal. 
Proof. By Green's identity

$$
T\left(D_{\varepsilon}\right)=2 \int_{D_{\varepsilon}} u_{\varepsilon}(y) d y=2 \int_{D} u(x, \varepsilon)|J(x, \varepsilon)| d x
$$

where the Jacobian

$$
J(x, \varepsilon)=\frac{\partial \phi(x, \varepsilon)}{\partial x}=1+\varepsilon \nabla \cdot S(x)+\varepsilon^{2} R(x, \varepsilon)
$$

with $R$ uniformly bounded for $x \in D$ and $\varepsilon$ sufficiently small. Thus by (2)

$$
\begin{aligned}
\left.\frac{d T\left(D_{\varepsilon}\right)}{d \varepsilon}\right|_{\varepsilon=0} & =2 \int_{D} u^{\prime}(x) d x+2 \int_{D} \nabla \cdot\left(u_{0} S\right) d x=-\int_{D}\left(\Delta u_{0}\right) u^{\prime} d x \\
& =-\int_{\partial D} \frac{\partial u_{0}}{\partial n} u^{\prime} d x=\int_{\partial D}\left(\frac{\partial u_{0}}{\partial n}\right)^{2}(S \cdot n) d s .
\end{aligned}
$$

We next consider electrostatic capacity. Let $D$ be a bounded Lipschitz domain in $\mathbf{R}^{n}$ having a boundary which consists of two simple closed surfaces $\Gamma$ and $\Gamma_{0}$ one of which encloses the other. Let $u$ be the solution of $\Delta u=0$ on $D, u=0$ on $\Gamma_{0}$ and $u=1$ on $\Gamma$. The electrostatic capacity of $D$ is defined by

$$
C(D)=\int_{\Gamma} \frac{\partial u}{\partial n} d s=\int_{D}|\nabla u|^{2} d x
$$

where $n$ is the outer normal. We consider variations in $C(D)$ when $\Gamma_{0}$ is varied while $\Gamma$ is fixed and assumed to be $C^{1, \delta}$, for some $\delta>0$.

We can generalize slightly by consider the problem $\Delta u=0$ on $D, u=0$ on $\Gamma_{0}$, and $u=f$ on $\Gamma$ where $f \in C^{1, \delta}(\bar{D})$. Again let $D_{\varepsilon}=\phi_{\varepsilon}(D)$ where $\phi_{\varepsilon}(x)=$ $x+\varepsilon S(x)$, and let $S$ be a Lipschitz vector field vanishing in a neighborhood of $\Gamma$. Let $u_{\varepsilon}$ be the solution of $\Delta u_{\varepsilon}=0$ on $D_{\varepsilon}, u_{\varepsilon}=f$ on $\Gamma, u_{\varepsilon}=0$ on $\Gamma_{\varepsilon}=\phi_{\varepsilon}\left(\Gamma_{0}\right)$. Let $u(x, \varepsilon)=u_{\varepsilon}\left(\phi_{\varepsilon}(x)\right)$ and let $u^{\prime}$ be defined by (1). Note that since $\Gamma$ is $C^{1, \delta}, \nabla u(x, \varepsilon)$ and $\nabla u^{\prime}(x)$ extend to continuous functions on $\Gamma$.

Theorem 3. Let $D$ be the domain between two simple closed surfaces $\Gamma$ and $\Gamma_{0}$ in $\mathbf{R}^{n}$, where $\Gamma_{0}$ is Lipschitz and $\Gamma$ is $C^{1, \delta}$. Define $u(x, \varepsilon)$ and $u^{\prime}(x)$ as in the previous paragraph. If $S$ vanishes in a neighborhood of $\Gamma$, then

$$
\lim _{\varepsilon \rightarrow 0} \frac{1}{\varepsilon}\left[\frac{\partial u}{\partial x_{i}}(x, \varepsilon)-\frac{\partial u}{\partial x_{i}}(x, 0)\right]=\frac{\partial u^{\prime}}{\partial x_{i}}(x)
$$

for all $x \in \Gamma$. This limit also holds in the sense of $L^{1}(\Gamma)$.

Corollary 4. Under the same hypotheses on $D$ as is Theorem 3

$$
\left.\frac{d C\left(D_{\varepsilon}\right)}{d \varepsilon}\right|_{\varepsilon=0}=-\int_{\Gamma_{0}}\left|\nabla u_{0}\right|^{2}(S \cdot n) d s,
$$

where $n$ is the outward normal.

Theorem 3 will be proved in Section 2. The corollary is an immediate consequence since

$$
\begin{aligned}
\left.\frac{d C\left(D_{\varepsilon}\right)}{d \varepsilon}\right|_{\varepsilon=0} & =\int_{\Gamma} \frac{\partial u^{\prime}}{\partial n} d s=\int_{\partial D} u_{0} \frac{\partial u^{\prime}}{\partial n} d s=\int_{\partial D} \frac{\partial u_{0}}{\partial n} u^{\prime} d s \\
& =-\int_{\Gamma_{0}}\left(\frac{\partial u_{0}}{\partial n}\right)^{2}(S \cdot n) d s .
\end{aligned}
$$




\section{Proofs}

Proof of Theorem 1. As in [8], define the differential operator $L_{\varepsilon}$ by

$$
L_{\varepsilon}=-\sum_{i, k} \frac{\partial}{\partial x_{i}}\left(A_{i k}(x, \varepsilon) \frac{\partial}{\partial x_{k}}\right)
$$

where

$$
A_{i k}=\sum_{j} J(x, \varepsilon) \frac{\partial x_{i}}{\partial x_{j}^{*}} \frac{\partial x_{k}}{\partial x_{j}^{*}},
$$

$x^{*}=\phi_{\varepsilon}(x)$ and $J(\cdot, \varepsilon)$ is the Jacobian of the transformation $\phi_{\varepsilon}$. Then $L_{\varepsilon}\left(v \circ \phi_{\varepsilon}\right)=-(\Delta v) \circ \phi_{\varepsilon}$. Since the function $\varepsilon \mapsto \partial A_{i k} / \partial \varepsilon$ is differentiable from $\left(-\varepsilon_{0}, \varepsilon_{0}\right)$ into $L^{\infty}(D)$ for $\varepsilon_{0}$ sufficiently small and all $i, k$, it follows that $\varepsilon \mapsto L_{\varepsilon}$ is differentiable into the set of bounded operators $B\left(H_{0}^{1}(D), H^{-1}(D)\right)$ with the operator topology, the derivative given by

$$
L_{\varepsilon}^{\prime}=-\sum_{i, k} \frac{\partial}{\partial x_{i}}\left(\frac{\partial}{\partial \varepsilon} A_{i k}(x, \varepsilon) \frac{\partial}{\partial x_{k}}\right) .
$$

Let $G_{\varepsilon}: H^{-1}(D) \rightarrow H_{0}^{1}(D)$ be the inverse of $L_{\varepsilon}$. Then $\varepsilon \mapsto G_{\varepsilon}$ is differentiable with $d G_{\varepsilon} / d \varepsilon=-G_{\varepsilon} L_{\varepsilon}^{\prime} G_{\varepsilon}$. Since $u(\cdot, \varepsilon)=G_{\varepsilon} f$, it follows that $\varepsilon \mapsto u(\cdot, \varepsilon)$ is differentiable into $H_{0}^{1}(D)$. Let $w$ be any solution to $-\Delta w=f$ on $D^{\prime}$ and let $v_{\varepsilon}=u_{\varepsilon}-w, v(\cdot, \varepsilon)=v_{\varepsilon} \circ \phi_{\varepsilon}$. Then $\varepsilon \mapsto v(\cdot, \varepsilon)$ is differentiable into $H^{1}(D)$ and $L_{\varepsilon} v(\cdot, \varepsilon)=0$. Therefore, $L_{\varepsilon}^{\prime} v(\cdot, \varepsilon)+L_{\varepsilon} \frac{d v(\cdot, \varepsilon)}{d \varepsilon}=0$ (in the sense of distributions). As in [8], $L_{0}^{\prime}\left(v_{0}\right)=\Delta\left(\nabla v_{0} \cdot S\right)$ (again in the sense of distributions), since $\Delta v_{0}=0$. Thus, letting

$$
\dot{v}=\left.\frac{d v(\cdot, \varepsilon)}{d \varepsilon}\right|_{\varepsilon=0}, \quad \dot{u}=\left.\frac{d u(\cdot, \varepsilon)}{d \varepsilon}\right|_{\varepsilon=0}
$$

we have $\Delta(\dot{v})=\Delta(\nabla v \cdot S)$. Therefore $\dot{v}=\nabla v \cdot S+h$ where $h$ is harmonic on $D$ and $\dot{u}=\dot{v}+\nabla w \cdot S$ implies $\dot{u}=\nabla u_{0} \cdot S+h$. On $\partial D, u(x, \varepsilon)=0$, for all $\varepsilon ;$ hence $h=-\nabla u_{0} \cdot S$ on $\partial D$, so $h=u^{\prime}$. This proves Theorem 1 .

Turning to the proof of Theorem 3 , let $\Gamma$ and $\Gamma_{0}$ be nonintersecting simple closed surfaces, one of which is inside the other, and let $D$ be the domain between $\Gamma$ and $\Gamma_{0}$. We assume that $\Gamma$ is $C^{1, \delta}$ and that $\Gamma_{0}$ is Lipschitz. Let $S$ be a vector field which vanishes in a neighborhood of $\Gamma$. Let $\Gamma_{1}$ be a $C^{1, \delta}$ surface between $\Gamma$ and $\Gamma_{0}$ such that $\operatorname{supp} S \subset D_{0}$, where $D_{0}$ is the region between $\Gamma_{1}$ and $\Gamma_{0}$. Let $D_{1} \subset D$ be an open set such that $\Gamma \subset \bar{D}_{1}$ and $\bar{D}_{1} \cap \bar{D}_{0}=\varnothing$. Let $D_{\varepsilon}=\phi_{\varepsilon}(x)$ where $\phi_{\varepsilon}(x)=x+\varepsilon S(x)$, and let $u_{\varepsilon}$ be the solution of $\Delta u_{\varepsilon}=0$ on $D_{\varepsilon}, u_{\varepsilon}=f$ on $\Gamma, u_{\varepsilon}=0$ on $\Gamma_{\varepsilon}=\phi_{\varepsilon}\left(\Gamma_{0}\right)$, where $f \in$ $C^{1, \delta}(\bar{D})$. Let $g_{\varepsilon}$ be the Green's function for $D_{\varepsilon}$ and let $u(x, \varepsilon)=u_{\varepsilon}\left(\phi_{\varepsilon}(x)\right)$ and $g(x, y, \varepsilon)=g_{\varepsilon}\left(\phi_{\varepsilon}(x), \phi_{\varepsilon}(y)\right)$.

Lemma 5. $g(\cdot, y, \varepsilon)$ and $\partial g / \partial y_{j}(\cdot, y, \varepsilon) \in H^{1}\left(D_{0}\right)$ with

$$
\left\|\partial g / \partial y_{j}(\cdot, y, \varepsilon)\right\|_{H^{1}\left(D_{0}\right)} \leq C \text { and }\|g(\cdot, y, \varepsilon)\|_{H^{1}\left(D_{0}\right)} \leq C
$$

where $C$ is independent of $y \in \bar{D}_{1}$ and $|\varepsilon|<\varepsilon_{0}$ for some $\varepsilon_{0}>0$ sufficiently small.

Proof. $\partial g_{\varepsilon} / \partial y_{j}(x, y)=0$ for $x \in \phi_{\varepsilon}\left(\Gamma_{0}\right)$ and $y \in \bar{D}_{1}$. Since $\Gamma_{1}=\phi_{\varepsilon}\left(\Gamma_{1}\right)$ is a $C^{1, \delta}$ curve and $\partial g_{\varepsilon} / \partial y_{j}(\cdot, y)$ is differentiable in a neighborhood of $\Gamma_{1}$, 
$\partial g_{\varepsilon} / \partial y_{j}(\cdot, y) \in H^{1}\left(\Gamma_{1}\right)$ and $\left\|\partial g_{\varepsilon} / \partial y_{j}(\cdot, y)\right\|_{H^{1}\left(\Gamma_{1}\right)} \leq C$ independent of $y \in$ $\bar{D}_{1}$ and small $\varepsilon$. There exists a $C$ such that $\|u\|_{H^{1}(D)} \leq C\|u\|_{H^{1}(\partial D)}$ for all functions $u$ harmonic on $D$ for which $\left.u\right|_{\partial D} \in H^{1}(\partial D)$ [11]. Therefore,

$$
\left\|\partial g_{\varepsilon} / \partial y_{j}(\cdot, y)\right\|_{H^{1}\left(\phi_{\varepsilon}\left(D_{0}\right)\right)} \leq C .
$$

Since $\left\|v \circ \phi_{\varepsilon}\right\|_{H^{1}\left(D_{0}\right)} \leq C\|v\|_{H^{1}\left(\phi_{\varepsilon}\left(D_{0}\right)\right)}$, it follows that $\left\|\partial g / \partial y_{j}(\cdot, y, \varepsilon)\right\|_{H^{1}\left(D_{0}\right)} \leq$ $C$. Similarly for $g$.

Proof of Theorem 3. By a theorem of Dahlberg [7], $D$ Lipschitz implies

$$
u(x, \varepsilon)=-\int_{\Gamma} \frac{\partial g}{\partial n_{y}}(x, y, \varepsilon) f(y) d s_{y}
$$

and

$$
u^{\prime}(x)=\int_{\Gamma_{0}}(S(w) \cdot n) \frac{\partial u_{0}}{\partial n}(w) \frac{\partial g}{\partial n_{w}}(x, w) d s_{w},
$$

where $u^{\prime}$ is defined by (1). We wish to justify differentiation of (6) under the integral sign with respect to $\varepsilon$ and $x$. By equation (2.4.28) of [8],

$$
\frac{\partial g}{\partial \varepsilon}(x, y, \varepsilon)=-\int_{D} \sum_{i, k} \frac{\partial A_{i k}}{\partial \varepsilon}(w, \varepsilon) \frac{\partial g}{\partial w_{i}}(w, x, \varepsilon) \frac{\partial g}{\partial w_{k}}(w, y, \varepsilon) d w,
$$

where $A_{i k}$ is defined by (4). It follows from the preceding lemma and Cauchy's inequality that

$$
\int_{D_{0}} \frac{\partial^{2} g}{\partial x_{j} \partial w_{i}}(w, x, \varepsilon) \frac{\partial^{2} g}{\partial y_{k} \partial w_{l}}(w, y, \varepsilon) d w \leq C
$$

where $C$ is independent of $x$ and $y \in \bar{D}_{1}$ and $\varepsilon$ sufficiently small. Noting that $\left|\partial A_{i l}(w, \varepsilon) / \partial \varepsilon\right| \leq C$, for all $w \in D, \varepsilon$ sufficiently small and all $i, l$ and that the integral in (8) can be taken over $D_{0}$, we see that (8) can be differentiated under the integral with respect to $x_{j}$ and $y_{k}$ and that

$$
\frac{\partial^{3} g}{\partial x_{j} \partial y_{k} \partial \varepsilon}(x, y, \varepsilon) \leq C
$$

where $C$ is independent of $x$ and $y \in \bar{D}_{1}$ and $\varepsilon$ sufficient small. It therefore follows that (6) can be differentiated under the integral sign with respect to $x_{j}$ and $\varepsilon$ and that

$$
\frac{\partial^{2} u}{\partial x_{j} \partial \varepsilon}(x, \varepsilon)=-\int_{\Gamma} \frac{\partial^{3} g}{\partial x_{j} \partial n_{y} \partial \varepsilon}(x, y, \varepsilon) f(y) d s_{y}
$$

for $x \in \bar{D}_{1}$. Since

$$
\frac{1}{\varepsilon}\left[\frac{\partial u}{\partial x_{i}}(x, \varepsilon)-\frac{\partial u}{\partial x_{i}}(x, 0)\right]=\frac{\partial^{2} u}{\partial x_{i} \partial \varepsilon}(x, \theta(x, \varepsilon) \varepsilon)
$$

where $0 \leq \theta(x, \varepsilon) \leq 1$ it follows from (9), (10) and the Lebesgue Dominated Convergence Theorem that

$$
\frac{1}{\varepsilon}\left[\frac{\partial u}{\partial x_{i}}(x, \varepsilon)-\frac{\partial u}{\partial x_{i}}(x, 0)\right] \rightarrow \frac{\partial^{2} u}{\partial x_{i} \partial \varepsilon}(x, 0)
$$

in $L^{1}(\Gamma)$ as well as pointwise as $\varepsilon \rightarrow 0$. 
Since $L_{\varepsilon} u_{\varepsilon}=0$, it follows that $L_{\varepsilon}^{\prime} u_{\varepsilon}+L_{\varepsilon} \frac{\partial u}{\partial \varepsilon}=0$. As in the proof of Theorem $1 L_{0}^{\prime} u_{0}=\Delta\left(\nabla u_{0} \cdot S\right)$, which implies that $\Delta(\dot{u})=\Delta\left(\nabla u_{0} \cdot S\right)$ where $\dot{u}$ is defined by (5). Since $u$ is independent of $\varepsilon$ on $\partial D$, it follows that $\dot{u}=\nabla u_{0} \cdot S+u^{\prime}$ where $u^{\prime}$ is harmonic on $D, u^{\prime}=-\nabla u_{0} \cdot S$ on $\partial D$. It follows from (9) and (10) that $\frac{\partial^{2} u}{\partial x_{j} \partial \varepsilon}$ is uniformly continuous on $D_{1} \times\left(-\varepsilon_{0}, \varepsilon_{0}\right)$. Therefore

$$
\left.\frac{\partial}{\partial \varepsilon}\left(\frac{\partial u_{\varepsilon}}{\partial x_{j}}\right)\right|_{\varepsilon=0}=\frac{\partial \dot{u}}{\partial x_{j}}=\frac{\partial}{\partial x_{j}}\left(\nabla u_{0} \cdot S\right)+\frac{\partial u^{\prime}}{\partial x_{j}}=\frac{\partial u^{\prime}}{\partial x_{j}} \quad \text { on } \Gamma
$$

since $S$ vanishes in a neighborhood of $\Gamma$. This completes the proof of Theorem 3.

By using the methods of Theorem 3, one can also give a proof of Theorem 1 that yields $(2)$ as a pointwise limit as well as a limit in $H_{0}^{1}(D)$. Although we omit the details, this can be done by using (8) and showing that $M(x, \cdot) \in$ $L_{\alpha}^{p}(D)$ for some $p>1$ and $\alpha>0,\|M(x, \cdot)\|_{L_{\alpha}^{p}(D)} \leq C$, where $M(x, y)=$ $\int_{D} \frac{\partial g}{\partial w_{i}}(w, x) \frac{\partial g}{\partial w_{k}}(w, y) d w$ and $C$ depends only on the Lipschitz constant of $D$. This can be established by making use of Theorem 1.1 of [12].

\section{Applications}

Variational formulas of the type derived above have applications in control theory (shape optimal design, see e.g. [6], [5], [15]), inverse problems, crack detection, and free boundary problems. For example one can consider a functional which arose in a recent study of "crack detection" by the first author. Suppose $D$ is a Lipschitz domain in $\mathbf{R}^{2}$ and we specify two disjoint arcs $E$ and $F$ on the boundary $\Gamma$. Consider the harmonic measure of $E$, that is, the function $u^{E}$ harmonic on $D$ with boundary values 1 on $E$ and 0 on $\partial D-E$, and define the functional

$$
C=\int_{F} \frac{\partial u^{E}}{\partial n} d s
$$

If we consider interior variations of the domain $D$ which leave $E$ and $F$ fixed, the methods of Theorem 3 can be used to show that the variation of $C$ is given by

$$
\left.\frac{d C}{d \varepsilon}\right|_{\varepsilon=0}=\int_{F} \frac{\partial u^{\prime}}{\partial n} d s=\int_{\Gamma}(S \cdot n) \frac{\partial u^{E}}{\partial n} \frac{\partial u^{F}}{\partial n} d s
$$

where $u^{\prime}$ is harmonic on $D$, and $u^{\prime}=-\nabla u^{E} \cdot S$ on $\Gamma$.

We conclude by considering a variational approach to Prandtl-Batchelor flows in the case of a bounded flow domain. We consider two simple closed curves in the plane, $\Gamma$ and $\gamma, \gamma$ contained in the bounded domain $\Omega$ bounded by $\Gamma$. Denote by $\Omega_{2}$ the domain bounded by $\gamma$ and $\Omega_{1}$ the annular domain bounded by $\Gamma$ and $\gamma$. For given $\mu<0$ and $\omega>0$ we consider functions $\psi_{1}$ and $\psi_{2}$ satisfying

$$
\begin{gathered}
\Delta \psi_{1}=0 \text { on } \Omega_{1}, \quad \psi_{1}=0 \text { on } \gamma, \quad \psi_{1}=\mu \text { on } \Gamma, \\
-\Delta \psi_{2}=\omega \text { on } \Omega_{2}, \quad \psi_{2}=0 \text { on } \gamma .
\end{gathered}
$$

We may think of $\psi_{1}$ and $\psi_{2}$ as stream functions of irrotational flow in $\Omega_{1}$ and a constant vorticity flow in $\Omega_{2}$. In the model proposed by Batchelor [2] for large Reynolds number limits of solutions of the steady Navier-Stokes equations, a flow of this type was hypothesized in which the square of the tangential velocity 
has a constant jump across $\gamma$, i.e. $\left|\nabla \psi_{1}\right|^{2}-\left|\nabla \psi_{2}\right|^{2}=\sigma$ for some constant $\sigma \geq 0$. This may be thought of as a free boundary problem in which $\gamma$ is to be determined. Keeping $\Gamma$ fixed, we define the functional

$$
I(\gamma)=\int_{\Omega_{1}}\left|\nabla \psi_{1}\right|^{2}-\omega \int_{\Omega_{2}} \psi_{1}-\sigma\left|\Omega_{2}\right|=\int_{\Omega_{1}}\left|\nabla \psi_{1}\right|^{2}-\int_{\Omega_{2}}\left|\nabla \psi_{2}\right|^{2}-\sigma\left|\Omega_{2}\right| .
$$

We assume that $\Gamma$ is $C^{1, \delta}$. A formal application of our results shows that $\delta I=\int_{\Gamma}\left(\left|\nabla \psi_{1}\right|^{2}-\left|\nabla \psi_{2}\right|^{2}-\sigma\right) \delta n$; so the free boundary condition is a natural boundary condition for extremizing $I$.

We consider the problem of minimizing $I$. The minimization will be taken over a class of curves which are boundaries of domains $\Omega_{2}$ which have, for some $\varepsilon>0$, the $\varepsilon$-cone property; i.e. for each $x \in \partial \Omega_{2}$ there is a direction such that for all $y \in B(x, \varepsilon) \cap \Omega_{2}$ the isosceles triangle with vertex $y$, angle $\varepsilon$ at $y$ and altitude $\varepsilon$ oriented in this direction is contained in $\Omega_{2}$. It is shown in [6] that this class of domains is compact in the sense that their characteristic functions form a compact set in $L^{2}(\Omega)$, where $\Omega$ is the interior of $\Gamma$. Further, domains in this class are Lipschitz and satisfy a uniform extension property with respect to $H^{1}$. This last property can be used to prove continuity of the functional $I$ on the topology induced by convergence of characteristic functions of domains $\Omega_{2}$ in $L^{2}(\Omega)$.

Some insight into what is possible for this problem can be obtained by considering the circularly symmetric case. Suppose for definiteness that $\Gamma$ and $\gamma$ are concentric circles with radii 1 and $\rho$ respectively. The symmetric version of the free boundary problem can be solved explicitly. Restrictions on $\omega$ and $\sigma$ are required in order that there be a solution. For example, if $\sigma=0$, then $\omega \geq 4 e|\mu|$ is required. In general there are two solutions; one provides a maximum and the other a minimum for $I$ with respect to radial variations. The minimum is assumed at the larger value of $\rho$, and $I$ approaches 0 and $+\infty$ as $\rho$ approaches 0 and 1 respectively. Therefore, in the general case it seems reasonable to look for a minimizer $\Omega_{1}$ among domains which "are not too small."

For a general $C^{1, \delta}$ curve $\Gamma$ we can now give a sufficient condition on the class of curves $\gamma$ which will guarantee existence of solutions to the variational problem. Suppose $f$ is a conformal map of $\Omega=$ interior of $\Gamma$ onto the unit disk $\{|w|<1\}$. Let $\rho$ be the value of the radius which provides a local maximum of $I^{\text {sym }}$, where $I^{\text {sym }}$ is the functional in the circularly symmetric case, and let $\psi_{1}^{\text {sym }}, \psi_{2}^{\text {sym }}$ be the corresponding circularly symmetric solutions of (11). There is a positive upper bound for $\left|f^{\prime}\right|$ on $\Omega_{2}=f^{-1}(B(0, \rho))$. By rescaling (obtaining a new curve $\Gamma$ ) we can assume this upper bound is 1 . Denote for the moment by $\psi_{1}, \psi_{2}$ the solutions of (11) with $\gamma=\gamma_{0}=f^{-1}(\{|w|=\rho\})$. Then, if the conformal transplant of $\psi_{2}^{\text {sym }}$ is denoted by $\widehat{\psi}_{2}$, we have, since $\Delta \widehat{\psi}_{2}=-\omega\left|f^{\prime}\right|^{2}$, that $\Delta\left(\psi_{2}-\widehat{\psi}_{2}\right)<0$. Then we can deduce that $\left|\nabla \psi_{2}\right| \geq\left|\nabla \widehat{\psi}_{2}\right|$ on $\gamma_{0}$. Now suppose that $\widetilde{\Omega}_{2} \supset \Omega_{2}$ is a subdomain of $\Omega$ bounded by a curve $\tilde{\gamma}$ which has a point $P$ in common with $\gamma_{0}$. We will show that $I$ is decreased by giving $\tilde{\gamma}$ a small exterior displacement away from $P$. In fact by the results of the previous sections

$$
d I /\left.d \varepsilon\right|_{\varepsilon=0}=\int_{\tilde{\gamma}}\left(\left|\nabla \widetilde{\psi}_{1}\right|^{2}-\left|\nabla \widetilde{\psi}_{2}\right|^{2}-\sigma\right) S \cdot n d s,
$$


where $S$ is a vector field supported in a small neighborhood of $P$. The maximum principal implies that $\left|\nabla \widetilde{\psi}_{1}\right|<\left|\nabla \psi_{1}\right|$ and $\left|\nabla \widetilde{\psi}_{2}\right| \geq\left|\nabla \psi_{2}\right|$ at $P$, so that

$$
\left|\nabla \widetilde{\psi}_{1}\right|^{2}-\left|\nabla \widetilde{\psi}_{2}\right|^{2}<\left|\nabla \psi_{1}\right|^{2}-\left|\nabla \psi_{2}\right|^{2} \leq\left|\nabla \widehat{\psi}_{1}\right|^{2}-\left|\nabla \widehat{\psi}_{2}\right|^{2}=\sigma\left|f^{\prime}\right|^{2} \leq \sigma
$$

making use of the fact that $\left|\nabla \psi_{1}^{\text {sym }}\right|^{2}-\left|\nabla \psi_{2}^{\text {sym }}\right|^{2}=\sigma$ by the choice of $\gamma_{0}$. We therefore consider minimization of $I$ over those curves $\gamma$ which satisfy an $\varepsilon$ cone condition and contain the above $\gamma_{0}$ in their interior. The above discussion shows that a possible minimizer is bounded away from $\gamma_{0}$. In a similar way a minimizer is bounded away from $\Gamma$. In fact the first term in $I$ is the capacity of the ring domain bounded by $\Gamma$ and $\gamma$ and it follows from Lemma I.6.1 of [13] that this term becomes infinite as $\gamma$ approaches $\Gamma$. It now follows from the compactness result of Chenais cited above that a minimizing curve $\gamma$ exists.

Unfortunately, we cannot deduce from (13) that $\left|\nabla \psi_{2}\right|^{2}-\left|\nabla \psi_{1}\right|^{2}-\sigma=0$ for the minimizing curve. The problem is that general displacements of the minimizer $\gamma$ do not remain in the class under which the minimization was taken if $\gamma$ does not in fact satisfy the cone property with some larger value of $\varepsilon$. This seems to us to be an inherent difficulty of the variational approach to this free boundary problem. Although we have been unable to resolve this difficulty, the partial results obtained are of value in an approximate solution procedure. One need only check that the minimizer obtained does not achieve the a priori restriction on $\varepsilon$ (i.e. the Lipschitz constant).

Some comment should be made at this point about the relationship of our result with the fundamental work of Alt, Caffarelli and Friedman [1] on variational problems in which the solution has a jump in $|\nabla \psi|^{2}$ across the free boundary. (We will refer to [1] in particular although these problems were dealt with in a series of papers.) In particular that work implies results for the problem of minimizing $J$ where $J(\psi)=\int_{\Omega}\left(|\nabla \psi|^{2}+Q(\psi)\right)$, with $\Omega$ bounded, $\psi$ in $H^{1}(\Omega)$ with $\psi$ prescribed on $\partial \Omega$, and $Q(\psi)$ taking different constant values for $\psi>0$, and $\psi<0$. For our purposes we may take these values to be 0 and $\sigma$ respectively. It is proven in [1] that a continuous solution exists; it is harmonic on $\{\psi=0\}$ and satisfies the jump condition across $\psi=0$ in an average sense (equation (2.2) of [1]). These are in the nature of preliminary results and the deep theorems given are concerned with regularity of the free boundary $\{\psi=0\}$. In particular it is shown that the free boundary is $C^{1}$ if $n=2$. (A result in $n$ dimensions was obtained later by Caffarelli [3] using his results on Harnack inequalities for free boundary problems.)

If $Q(\psi)$ is modified for $\psi>0$ to $Q(\psi)=-2 \omega \psi$, then a formal solution of the variational problem satisfies $-\Delta \psi=\omega$ when $\psi>0$, and a variational problem corresponding to the Prandtl-Batchelor problem is obtained. The resulting functional $J$ is essentially an extension of the functional $I$ defined above, since $I(\gamma)=J(\psi)+c$, where $\left.\psi\right|_{\Omega_{i}}=\psi_{i}, \Omega=\Omega_{1} \cup \Omega_{2}$ and $c=-\sigma|\Omega|$. Those results of [1] that we referred to as preliminary can be rather easily generalized to this modified $J$. In particular existence of a solution, its continuity, satisfaction of the Laplace and Poisson equations off the free boundary and a limiting relation analogous to equation (2.2) of [1] can be proven. However, the deeper regularity results for the free boundary are more difficult. Having Poisson's equation, rather than Laplace's equation, on the region where $\psi>0$ not only complicates the proof of regularity, but also certain properties of the free boundary do not carry over to this case. In [1] global properties of the 
free boundary are obtained from the fact that both of the sets $\{\psi>0\}$ and $\{\psi<0\}$ are connected to the boundary (section 8 of [1]). However, for the Prandtl-Batchelor problem the argument establishing this does not hold for the set $\{\psi>0\}$. If, as in our problem, the prescribed value of $\psi$ on $\partial \Omega$ is $\mu<0$, we may expect that $\{\psi>0\}$ is bounded away from $\partial \Omega$, but there is no a priori reason to expect that this set is connected. We conjecture that it is not in general. We also note that for sufficiently small values of $\sigma$ and $\omega$ the functional $J$ will have as minimizer the constant function $\psi=\mu$, in which case $\{\psi>0\}$ is empty and no solution to the Prandtl-Batchelor problem is obtained. Analysis of the special case of the circular disk also indicates that for some values of $\sigma$ and $\omega$ a solution of the Prandtl-Batchelor problem may exist, even though the global minimizer of $J$ is the constant function $\psi=\mu$. Our previous discussion indicates a possible way of obtaining the solution of the Prandtl-Batchelor problem as a local minimizer in the context of our functional I.

The authors are indebted to David Jerison for informing us how the results of [12] can be used as indicated at the end of Section 3. The authors are grateful for the careful and thoughtful comments of the referee which led to substantial improvements over a first version of this paper.

\section{REFERENCES}

1. H. W. Alt, L. A. Caffarelli, and A. Friedman, Variational problems with two phases and their free boundaries, Trans. Amer. Math. Soc. 282 (1984), 431-461.

2. G. K. Batchelor, A proposal concerning laminar wakes behind bluff bodies at large Reynolds number, J. Fluid Mech. 1 (1956), 388-398.

3. L. A. Caffarelli, A Harnack inequality approach to the regularity of free boundaries. Part II: Flat free boundaries are Lipschitz, Comm. Pure Appl. Math. 52 (1989), 55-78.

4. R. Caflisch, Mathematical analysis of vortex dynamics, Proc. of the Workshop on Mathematical Aspects of Vortex Dynamics, (R. Caflisch, ed.), SIAM, Philadelphia, PA, 1989, pp. 1-24.

5. J. Cea, Problems in shape optimal design, Optimization of Distributed Parameter Structures, II (E. Haig and J. Cea, eds.), Sijthoff \& Noordhoff, Rockville, MD, 1981, pp. 1005-1047.

6. D. Chenais, On the existence of a solution in a domain identification problem, J. Math. Anal. Appl. 52 (1975), 189-219.

7. B. Dahlberg, On estimates of harmonic measure, Arch. Rational Mech. Anal. 65 (1977), 275-288.

8. P. R. Garabedian and M. Schiffer, Convexity of domain functionals, J. Analyse Math. 2 (1953), 281-368.

9. J. Hadamard, Memoire sur le probleme d'analyse relatif à l'equiliöre des plaques élastiques encastrées (1908), Oeuvres de J. Hadamard, C.N.R.S., Paris, 1968.

10. D. S. Jerison, Prescribing harmonic measure on convex domains, Invent. Math. 105 (1991), 375-400.

11. D. S. Jerison and C. E. Kenig, Boundary value problems on Lipschitz domains, Studies in Partial Differential Equations (W. Littmann, ed.), MAA Studies in Math., vol. 23, MAA, Washington, D.C., 1982, pp. 1-68.

12. _ The inhomogeneous Dirichlet problem in Lipschitz domains, preprint.

13. O. Lehto and K. I. Virtanen, Quasiconformal mappings in the plane, Springer-Verlag, New York, 1973. 
14. J. W. Rayleigh, The theory of sound, 2nd ed., Cambridge Univ. Press, 1894-1896.

15. J. Simon, Differentiation with respect to the domain in boundary value problems, Numer. Funct. Anal. Optim. 2 (1980), 649-687.

Department of Mathematics, Wichita State University, Wichita, Kansas 67260

E-mail address: arelcrat@twsuvm.uc.twsu.edu

E-mail address: miller@twsuvm.uc.twsu.edu 